The senile form is, to my mind, essentially degenerative, and may therefore, in my opinion, be the result of various factors, acting alone or in combination. Among these is, no doubt, to be reckoned the ordinary type of arthritis deformans, which may pass on into it, just as the large white kidney may - somewhat rarely perhaps-undergo contraction and fibrosis. In many instances there is no precedent arthritis deformans, and in such cases we have to look to other etiological agencies, in particular old age, as the causes of degeneration. ${ }^{9}$ Sometimes it supervenes upon the chronic joint condition resulting from repeated attacks of acute rheumatism ; in fact, it may be almost laid down as an axiom that any diseased joint will, if the patient live long enough, become the seat of senile osteoarthritis.

Finally, the disease described by Dr. Still is an infective process which appears to be in no way related to any of the other forms.

REFERENCES.

1 Medical Annual, 1903-4. 2 Guy's Hospital Reports, vol. Ivii. 3 Trans. Royal Med.-Chir Society. vol. Ixxx. I Med. Record, April 18th. 1903. 5 Unters-ib. d. anat. Zust. d. Gelenke, etc., Berlin, 1885. 6 Deutsch. Zeitschr. f. Chirurgie, Ir. ' Virchow' Archiv, vol lv, and other papers. B Edn. Medical Jownal, January, 1903.
vol. vii, part 5 .

\section{THE MEDICAL TREATMENT OF INSANITY.}

By ROBERT JONES, M.D., B.S., F.R.C.S.ENG.,

Resident Physician and Superintendent, London County Asylum, Claybury.

THF great aim of medical art is the cure of disease, and in fulfiling this there are certain general principles which commend themselves to every medical practitioner, quite apart from the specialized treatment which is applied to the various maladies described in detail in the many textbooks upon medicine. These principles are, in short, hygienic, prophylactic, and directly remedial or therapeutic, and they apply to the treatment of insanity with even greater force than they do to diseases in general, for insanity results from neglect of the rules of health, and through attempts made to over-ride or to disregard the laws of Nature.

In hygienics we have the study of conditions which are of immense importance to the welfare of the whole population, such as those relating to occupations, to the density of the population, to drainage, air, and ventilation, to the character of the water and of food, as also those relating to personal and general cleanliness. The neglect of the laws of health deduced from these studies involves a lowered vigour and vitality, a diminished power of resistance to disease, and a general deterioration, if not a degeneration of both mental and bodily energy, the output of work in consequence is diminished, and any given stress approaches nearer the breaking point. Hygienic measures are of so much importance that the State now recognizes the necessity for the undivided attention of medical men and others in carrying them out, and public bodies find their highest function in establishing and perfecting conditions favouring a high standard of health among a community.

Prophylactic or preventive treatment implies that there are circumstances exposure to which tends to impair the general health, either directly or through tendencies derived from parents. Questions relating to alcohol and to immorality agitate the public mind from time to time in regard to insanity, as also in regard to vice, poverty, and crime; and measures considered for their control come under the designation of preventive treatment. Steps taken to prohibit the ale of alcoholic liquor are prophylactic measures in regard to insanity, as are also those relating to the marriage of neurotic and insane persons, which in the opinion of many should be prohibited. The question of betrothals and their suspension is too long and complicated a problem to. be discussed here, but this special aspect has recently been before the public in the effort made to establish the science of Eugenics or stirpiculture - the propagation of the most fit-which is the direct and conscious application to man of the law of natural selection. Further preventive measures may be considered in the dissemination of knowledge as to the common causes of insanity and the means to be adopted for its avoidance, more especially by the treatment of incipient symptoms of mental disease in the out-patient departments of our hospitals, where prompt and skilful advice would be given, also by assistance or employment given to those who have recovered from mental diseases, as many of these not only fail to find work, but also suffer much hardship, which not infrequently causes them to relapse.
What should be done when a person not previously insane is threatened with symptoms of mental disorder? In many cases the treatment is obvious. The exciting cause, if any, must be removed, over-work must cease, overworry must be controlled, and anxiety and over-excitementin regard to religious matters, for example, to take a subject which at the present moment is commanding public attention-should be stopped; undue devotion must be restrained, and life generally must be on a less exciting and stimulating plane.

$A s$ to the remedial and therapeutic treatment of insanitywhich is the theme of our lecture-we are confronted with the portentous fact that on January 18t, 1904, there were 117, 199 persons, that is, I to every 288 of the population, who were certified or registered as insane in England and Wales and speaking generally, these were incarcerated under about 126,000 certificates (private patients necessitating two for their detention) a number which amounts to more than five certificates for every registered medical practitioner; to snch, therefore, this subject must be of much interest and importance. We also know that possibly an equal number of persons are on the borderland of certification, and that out of this group fresh cases are being daily precipitated into those of which the State-through the Lord Chancellor and the Lunacy Commissioners-has cognizance.

In the acute stage of no disease is care and attention more necessary, nor more expensive, nor, may it also be said, is there more hope of cure than there is in the early stages of acute insanity. Unfortunately, however, the study of . insanity is a long and painful experience ; it is also as interesting in theory as it is trying and exacting in practice.

The causes of insanity are, again, so complex; both so direct and so indirect and numerous; the "tissue of mind" is so complicated, its anatomy, physiology, and chemistry are so little understood; furthermore. the very nature of mind itself is so incomprehensible and mysterious that our treatment of insanity not infrequently amounts-as is also the case with many other diseases - to the treatment of symptoms as they arise-that is, to maintaining the strength of the patient by judicious nursing, to quieting hyperaesthetic senses, to raising the hopes and spirits of the despondent, and to promoting their general comfort. Possibly in no disease is it more necessary to support the patient's strength by appropriate nourishment, for the abnormal rapidity of disintegration which occurs in acute insanity impairs nutrition so quickly that death from exhaustion often takes place before the delirium subsides. It becomes imperative, therefore, to obviate this most frequent cause of death by a vigorous administration of un-irritating food, easy of digestion and nourishing, to be given frequently and in small quantities-"little and often." Foods, such as raw meat, or beef juice, peptonized beef tea, the yolk of egge, milk with soda water, or with biscuit powder, barley water, gruel, rice water, broths, arrowroot, cornflour, or Mellin's food, all these being eminently suitable-possibly also (if exhaustion and delirium are combined) dry wines, but meat juices especially-that is, if the patient takes exercise out of doors. It is most necessary in all cases of acute insanity (acute delirious mania, acute puerperal mania, acute restless melancholia, etc.) to supply-even forcibly with the tube and funnel, by the month or nose, two or three times daily, at fixed intervals-alimentary material to counteract the dangerous profound exhaustion, and also to renovate and maintain power; for, unless sustaining treatment is secured, the exhaustion may prove fatal in a few hours.

The judicious selection of food material is also important in regard to sleep. Loss of sleep is one of the most distressing and prevalent symptoms in the early stages of insanity. In more than one case of puerperal insomnia I have known an attack of insanity to be prevented by treating such secondary conditions as relieving pain, moderating uneasiness, allaying anxiety of mind, snothing irritability and inducing sleep ; for absolute repose is necessary and must be obtained by the use of drugs.

Whilst condemning the excessive use of calmatives and hypnotics as unjustifiable in the treatment of insanity, I must express $\mathrm{my}$ belief in sedatives. The motor excitement in certain forms of insanity-possibly from the production of a special toxin-is so fatiguing as well as $\epsilon$ xhausting, and the relief is so marked by the action of certain drugs, that in exceptional instances the morbid phenomena constituting insanity are cut short by the action of sedatives. Whilst discountenancing their use by persons who find in them a temporary alleviation to relieve sleeplessness, and who, more- 
over, in many instances have established a habit which may become a disease; yet it is not improbable that many persons have been saved from attacks of actual insanity by early treatment of this distressing symptom. It has been maintained that theacute insanities are of ten theresult of intraneuronic and extra-neuronic toxins which act injuriously upon the nerve centres, and that the sedative effect of drugs is antagonized by these. It certainly is a fact that long continued administration of drugs, such as sulphonal and the bromides, do require increasing doses to produce a given effect, which tends to show that diseased or damaged tissue is not so readily acted upon by sedatives, and that a larger dose is required by the insane than the normal person. Furthermore, a state of dementia may be brought about through the noxious effects of these drugg upon certain of the cortical neurons. We know that different parts of the nervous system are acted upon differently by various substances-for example, conium, curare, strychnine, the bromides, etc.-but our knowledge of the reason is unknown, and of their action empirical. We also know that restoration or anabolism takes place mostly during rest and sleep, and that in health action and reaction are always equal and contrary. In insanity there is probably some alteration, congenital or acquired, in the rhythm of waste and repair in the higher centres of the nervous system; but these centres, by the influence of sedatives, can be placed in a condition favourable to recuperation, and it is rather the combination of these sedatives with other remedies of a more general character which yield the best results-those, for instance, which favour nutrition by diminishing the irritability of the stomach or which assist its normal functions, such as pancreatin, pepsin, hydrochloric acid or papain, and those which also assist in the elimination of poisons from the skin, lungs, kidneys, and the alimentary canal, that is, which act upon the emunctory organs, as well as the organs of assimilation.

The first consideration, therefore, in regard to the treatment of insanity is to ascertain if there be any abnormal condition of the bodily organs, removal of which will favour the disordered brain functions; and instances are not infrequent -and I refer to the adolescent forms of insanity in anaemic girls - when the restoration of bodily organs to their normal functions is followed by mental improvement. The reestablishment of menstruation and the cure of anaemia mean in these cases mental recovery, indicating the necessity for the combination of sedatives with general remedies which help to restore and build up the various systems of the somatic whole.

Before approaching the pharmaceutical armamentarium I may point out that conduct which is violent, destructive, and filthy in habits, usually determines the question of restraint but if such conduct occurs in puerperal women, and shortly after confinement, or with fevers, accidents, or the onset of inflammation of important internal organs, or if it be due to alcohol, or to some known toxin; again, if it occurs in young persons, or it is sudden and possibly due to some one great overwhelming emotional disturbance-in these cases the physician and the friends must be iolerant, and the person treated at home as long as possible, or for at least a period of six weeks. On the other hand, if the patient be acutely suicidal or homicidal, or if the case be one of general paralysis with violence, seclusion under a certificate is then not only desirable, but necessary and obligatory.

In my opinion no supervision can equal the treatment given in our best private asylums and mental hospitals, which are especially constracted institations where the comforts of a home are fully provided, where there are no wide departures from customary surroundings upon which sensitive persons are so dependent for their happiness, and where a skilful and trained staff exercise the closest vigilance and supervision. In these homes individual treatment is carried out, and the great benefit to be derived from this direct influence cannot be over-estimated. Should there ke, however, as there often is, much feeling against certification, then residence as a voluntary boarder in a mental hospital can be arranged, but should there be an insurmountable dread of all institutions on the part of both the patient and his friends, then the best must be made of an improvised plan of private treatment and the patient will have to be provided for in a private house, preferably in the country-for walking in the open air is necessary - and the rooms should be on the ground floor, all risks from stairs, windows, cutting instruments, strings or cords, should be fully safeguarded against, as also risks from fire and water. At least two competent mental nurses, one for daks and the other for night duty, should be engaged, and they should make a daily written report of the pulse, temperature, food consumed, excretions, medicines taken, the hours of sleep, exercise out of doors, and all new symptoms. Relatives as nurses are less to be dreaded in cases of melancholia than in cases of mania, but usually in both conditions it is much better to engage competent nurses.

The treatment of mental disorder which comes within the experience of the general practitioner resolves itself most often into the treatment of cases of acute excitement or uncontrollable fury, but it must not be taken for granted that insanity with excitement invariably means a condition requiring antiphlogistic remedies. In cases in whom there is delirium, when the pulse is jull, bounding and firm, and there are symptoms of sthenic.inflammation, then venesection may prove beneficial. I have only used it for the venous engorgement of the status epilepticus, and then with good results. I have used the wine of tartarated antimony for furious excitement, with vascular and cerebral congestion, and when combined with morphine it is very effective. Aconite is a useful remedy in sthenic maniacal states and the exacerbations of recurrent insanity with cerebral hyperaemia. I have also used icebags to the head, and continuous immersion.

The difficulty. in keeping maniacal patients in a warm bath at the body temperature, or at $100^{\circ}$. has been so great that to avoid constant struggling I have abandoned the practice. The exact physiological effects and the therapeutic value of long immersion are not quite understood. It has been considered to be "derivative,"-that is, the cutaneous vessels dilate and thus lessen the quantity of blood in the nervous system, but it has also been suggested by some that the effect of warm baths is to increase metabolism, by others that the pressure of the water on the skin gives a tone to the cutaneous and to the general circulation which was previously deficient.

The use of the "wet pack" has been urged in cases of insanity with excitement, but there are dangers accompanying it, and I do not advocate it as a reliable remedy, although for certain bodily conditions accompanying insanity I have tried it witir good effect. Insanity, even with excitement, is a disease generally of under-nutrition, and whatever the pathological conditions may be which cause acute mania or acute melancholia, we can at present go no further than vaguely describe them as "a disturbed bio-chemical state" of the brain tissue, and my experience in the case of all the acute insanities recommends a generous and supporting rather than a depletory method of treatment.

In the treatment by the medical practitioner of cases of melancholia the tendency to suicide must be regarded as ever present, and the question of " foreign travel " not infrequently comes up for consideration. Speaking physiologically, the best brain work is done when the cortex receives abundant and definite stimuli from without. In travel, especially in the case of sea voyages, the life on board ship is exceedingly irksome, dull and monotonous, and should not be advised for cases of incipient insanity or even for cases in whom there may be a threatening of mental breakdown with symptoms of depression. In a voyage to Colombo made by a distinguished writer, physician, and man of affairs he mentions two cases, both of whom threw themselves overboard, whose lives would probably have been gpared had they not been urged to travel abroad. Even trips to the Continent do not provide an Englishman with the comforts he obtains at home, and which he hopes to receive abroad.

Electricity, administered in the form of currents through water in the form of electric baths, has been greatly advocated for the depressed varieties of insanity, more especially for atonic stuporose states in young persons. The galvanic current is passed through warm water at $100^{\circ}$, in which the patient is immersed. I have used such for many weeks at a time, daily, or several times a week, for about 10 to 30 minutes, and have kept a record of their weight whilst under treatment, and found it to go up, and their general mental and hodily conditions to improve. I confess to being favourably disposed to this form of electric stimulation, and it certainly appears to aid metabolism, although how and in what method I am unable to explain.

As to the action of drugg " upon the brain, it cannot be localized so accurately as can that of drugs acting on the spinal cord and nerves, but their action illustrates two important general laws-firstly, the law of dissolution, which shows that when a drug affects functions progressively, those first affected are the highest in development - that is to say, they are the last acquired by the individual and the last to appear in the species. The next affected are those next to 
the highest, and so on, until finally the lowest of all, from an evolutionary point of view-namely, the functions of respiration and circulation-are affected. We are acquainted with this exemplification in the case of alcohol, for the first functions to be disordered are those of the intellect, especially the highest, such as judgement and reason; then follow disorders of movement, and death from failure of respiration and circulation. Secondly, drugs in moderate doses excite a function but in large doses they paralyse it. This is familiarly illustrated in cases of chloroform inhalation, the first effects being motor excitement followed by motor paralysis, the excited limbs becoming motionless and weak. In the same manner drugs which are cerebral stimulants mav become hypnotics, as is exemplified in the use of opium. Conium is also one of the remedies which appears to have contradictory effects, but this is probably explained by the fact that the relation between conia and methyl conia-two alkaloids with opposite effects-varies in each preparation.

It is an excellent remedy as succus conii ( $m \times$ to $3 j$ ) for controlling motor excitement. With the abolition of abnormal muscular action the ideas become less rapid and mobile, mental processes become clear, and sleep occurs. It is best to begin with small doses, and to combine these with strychnine or some other cardiac stimulant. As a nervous tonic for persons who suffer from overwork, or who are run down, who are weary and easily tired, the various preparations of phosphorus, more especially, perhaps, the glycero-phosphates, have in certain quarters had a reputation. On theoretical grounds phosphorus should have been in much favour as a brain tonic, and it has been recommended for its efficacy in various forms of insanity; but the aphorism"without phosphorus no thought." is not borne out by the experience of the clinical psychiatrist.

The chemistry of the brain points out that lecithin, which enters largely into the structure of nervous tissue, is a compound which breaks up into glycero-phosphoric acid and cholin or neurin, and in certain bodily states associated with convulsions it appears probable that the amount of cholin in the blood represents the amount of waste caused by the breaking up of nervous material in the discharge of energy. But the particular rôle of phosphorus in the brain has hitherto received no satisfactory explanation, and, although the restorative nutrition of the brain is an important task for the psychiatrist, the problem of furnishing a reliable and assimilable preparation of phosphorus has yet to be solved; in consequence, the various phosphates may be administered ad nauseam without modifying illusions, hallucinations or delusions, and it is safe to assert that phosphoras is not to the brain in insanity what iron is to the blood in anaemia.

As to hypnotics, for the pure relief of insomnia paraldehyde in doses $3 \mathrm{ss}$ to $3 \mathrm{ij}$ (twice the amount is given for an enema) is one of the safest, for it stimulates cardiac action ; moreover, valvular heart disease is not a strong contraindication. It has a peculiarly disagreeable taste and smell, which are best disguised in highly-flavoured syrups or wine. It is not recommended in phthisis or bronchial affections, as, being volatile, it is mostly eliminated by the lungs. Amylene hydrate, in doses from $m x$ to $m \times x x$, has been much recommended, and I have used it. Somnal, gr. $x x$ to $\mathrm{gr}$. $\times x x$; methylal, $3 \mathrm{j}$ to $3 \mathrm{ij}$; hypnone, chloralamide, chloralose, hypnal, tetronal, and trional, have also their advocates. I have used sulphonal, gr. xx, three times a day (insoluble in water), trional (less insoluble), and tetronal. These have a cumulative effect which is their best quality, and they are excellent remedies for strong, dangerous, and aggressive maniacal patients. The effects of sulphonal do not appear fully for some hours, and as a night draught it should therefore be given early in the afternoon. The symptoms to be watched for are vertigo ataxia, and haematoporphyrinuria. After death the blood appears to be fluid, and to resemble dark port wine. The crystals may disturb digestion and cause diarrhoea. If the patient is kept in bed during the administration, the physiological effects are intensified. Hyoscine in subcutaneous

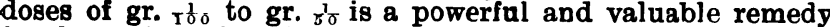
for the control of acute motor excitement in violent maniacal cases, those who are strong and muscular, and who in their fury may commit.homicide. It differs from sulphonal and trional in that its effects are immediate, but they soon pass off. When the motor disturbances are controlled, quiet sleep is produced. It is not a remedy for feeble cases. Hyoscyamine, dose gr. ${ }_{6}^{\prime}$, combined with morphine, is a very effective remedy for excitement and sleeplessness. Cannabis indica is uncertain in its action; it gives rise to visual hallu- cinations and rapidity of ideas, the latter causing a feeling in the mind of indefinite stretchts of time. When combined with bromide of potassium it is a useful remedy in senile insomnia, and especially when there is mental depression.

Possibly, of all the remedies for insomnia, the bromides are the most useful, for they lower reflex excitability and tend to slow down or diminish cortical activity. They are better hypnotics for states of excitement than for depression-but they may be combined with stimulants in such cases-and are therefore better suited for the insomnia of maniacal patients and for hallucinations of a tactile nature-paraesthetic states, as they are called-ocsurring in paretic and tabetic persons; also in cases of pharyngeal and laryngeal discomfort, giving rise to delusions of things in the throat. The effect ot a single large dose is much becter than that of long-continued under-dosing, and there is no danger of establishing a habit in regard to it. For alcoholic cases, for uterine reflex pains, such as occipital headaches, or in cases of neurasthenia with night terrors or unpleasant dreams, and combined with citrate of caffeine in cases of climacteric mental restlessness, the bromides are most useful. The only contraindication is unsoundness of the circulatory organs and anaemia, and the result of long-continued administration of bromides is a dull torpor, with loss of memory and stupor. Bromide of strontium is stated to be a better remedy for epilepsy than bromide of potassium, and to be also safer against cardiac failure, which is apt to occur in epilepsy, and bromide of sodium is stated to be the least often accompanied by gastric irritability. In association with chloral it is an excellent hypnotic for acute hallucinatory excitement, and possibly one of the best formulae is chloral hydrate, sodium bromide, and tincture of hyoscyamus. The contraindications to chloral are valvular or fatty heart disease, also if long administered with the bromides for epilepsy the tendency to cardiac failure from congestion arising in repeated fits should be remembered, also it loses effect by repetition. The dose of chloral is $\mathrm{gr}$. $x$ to $x x$, largely diluted and flavoured with aromatic syrups. It may also be given freely in milk. If given per rectum il is neceseary to compress with the hand to retain the enema. The bromides are administered in doses of gr. $x \times$ to $3 j$, or even up to $3 j$ and more. It is stated that neurotic medicines, such as are the bromides and also chloral, are best administered on an alkalinized stomach, hence a glass of milk is considered an effective adjuvant to such remedies. Possibly of all the hypnotics, opium together with its derivative morphine, is the one most often used, and it is probably the best remedy for psychic as well as physical pains, such also as are due to painful "vagus " sensations from cardiac crises and dyspnoea. Opium relieves acute mental depression, especially in old people, and it appears to act as a direct stimulant in the cardiac weakness of some senile cases. It appears to act directly also upon certain mental states, and to be antithetic to the painfully emotional states of melancholia and persecutory delusions. It is, in my opinion, contraindicated in states of mania, but morphine may then be administered. Morphine is often the best anodyne for the precordial pain of melancholia, as also for painful hallucinations in exhausted cases. A combination of opium with belladonna may assist to prevent the impairment of digestion caused by opium taken alone, especially in regard to constipation. The great danger in the use of opium is the toleration established and the risk there is in commencing a habit which may become a terrible diseare.

In contrast to hypnotics there are drugs which act upon the nervous system as sedatives, but stopping short of the actual hypnotic effects just described. Smaller doses of the bypnotic class effect this, but drugs such as valerian in large doses are known to control the agitation of neurasthenia or cases suffering from temporary functional disturbances, such as are seen and described as hysterical insanity. Camphor monobromate in gr. $v$ doses has been used with benefit in cases of insanity with epilepsy, also in spasmodic excitement or mental disturbances accompanying chorea. Lupulin has also its advocates for cases of neurasthenia and bysteiia, and the extract of Calabar bean in general paralysis of the insane. The extractum physostigmatis was at one time much in favour, upon the recommendation of Fraser. I may be allowed to mention as a possible remedy for this disease the use of thorium hydroxide, a heavy white powder possessing radio-active properties. I have used 500 grammes of the powder in a cap over the head, worn day and night, and two cases nave recovered after such treatment. It has been suggested that at the synapses-the arborization of the dentrites - there may be some radio-active body which either 
originates, transmits, or intensifies nervous currents. If its possible modification of this disease is advantageously affected by thorium hydroxide the benefit may thus be explained. Possibly no direct effect is obtained, but at any rate it is worth a trial, and something is being done for this hitherto incurable condition. In the congestive vertigo of this dif ease the fluid extract of ergot has been tried, upon the theory that it acts as a vascular stimulant upon the vasomotor centre, causing a contraction of the cerebral arterioles. It has been recommended to be given every few hours during the day, and alternating with bromide at night. For the seizures occurring in epilepsy, general paralysis, and puerperal insanity I have used chloroform inhalation and chloral, but prefer the latter.

I have used ergot for insanity about the climacteric period associated with menorrhagia. There are certain headaches and neuralgias about the "change of life" which almost incapacitate the sufferer from social and domestic duties. Those so-called lancinating pains may be associated with mental restlessness and actual insanity, and no remedy seems to be of permanently good effect. I have used antipyrin as an analgesic in such cases in doses from gr, $v$ to gr, $x x$, but care is needed from the depressing cardiac effect, and antipyrin always needs a stimulant to follow. Phenacetin gr. $x$ with quininae sulph. gr. $j$ has proved a useful remedy, as also the citrate of caffeine in gr. ijj doses. The peculiar restlessness of mental depression in regard to which there is no definite pathology, other than that it appears to have its basis in a summation of the organic sensations-and presumably caused by pneumogastric enervation -is often benefited by citrate of caffeine associated with small repeated doses of opium. It is in such cases that erythroxylon coca has been so much used, possibly also it js in similar cases that alcohol fulfils a definite therapeutic rôle, but it is necessary to beware of initiating a pernicious habit. As to alcohol, in the great majority of cases of insanity it is not essential, but I do not hesitate to use it in small doses every few hours in the exhaustion of acute mania, more especially in the puerperal form of insanity. It often interferes with digestion, and it is always best administered with liquid nourishment-either with milk, soup, beef tea, or broth. In acute insanity it is often difficult to get the patient to take solid food, but by coaxing he may be persuaded to take fluids, and alcohol may thus be effectively combined with it. The physiological effects of alcohol can be demonstrated by the sphygmograph. Alcohol increases the force and frequency of the beart's action and stimulates vascular tone, but in large doses alcohol acts as a definite depressant. Owing to this physiological action it is a dangerous remedy in the various forms of mental depression, for it gives the feeling of buoyancy and stimulation without any lasting benefit and without the sustaining action of food. In the excitement of mania the skin acts more freely as an eliminating organ than it does in depressed mental states, and more alcohol can therefore be tolerated in cases of mania. Strychnine gr. $\frac{1}{30}$ subcutaneously injected and digitalis in $m x$ doses every hour for three hours and then stopping are useful in cases of exhaustion from acute insanity, also in cases of cerebral anaemia as a cardiac stimulant, especially when forced feeding is being adopted. Strychnine affects the whole nervous centres and not the gpinal cord only. Nux vomica $m v$ may be used with much good effect in the convalescing stages as a general tonic. The prolonged use of strychnine or nux vomica is ineffective, and they are better given alternately for a few weeks and with about one week's interval.

In the earlier part of my paper I have referred to eliminating medicines. The chief eliminating organs are the skin, the lungs, the kidneys, and the bowels. In no morbid condition is it more important to clear the prima via than it is in insanity. Laxatives and purgatives are absolutely necessary, and in free doses-usually twice the amount required by a healthy person. Depressed mental states, irritability, and even the convulsive seizures in cases of general paralysis or epilepsy are often due to auto-intoxication caused by constipation, and consequent absorption of poisons from the alimentary canal. Constipation, indeed, is the bane of insane persons, and podophyllin, elaterium, colocynth calomel, and at times even croton oil, are necessary in full doses to cause a free action of the bowels.

The hydragogue cathartics can be freely administered in cases of insanity, and "house mixture "-the pharmacopoeial hst. sennae co. - is a safe and salutary corrective in such cases, and may be systematically administered once or twice a week with much benefit. The action of the skin and sidneys is assisted by the administration of diuretics and diaph ( retics, but the best of medicines is exercise out of doors, which causes the langs and skin to act freely and naturally, and which facilitates the exudation of substances noxious to healthy mental action.

It is hardly necessary in such a paper as this, and in addressing such readers, to emphasize the study of the various diatheses, to know our patient with his various idiosyncrasies -namely, his tendency to gout, rheumatism, anaemia, renal or cardiac disease, phthisis, myxoedema, ague, or syphilitic lesions, which all require special treatment. The question of organo-therapy and serum-therapeutics is also too wide to be discussed within the compass of a lecture such as this, but I will summarize my suggestions by recommending in cases of sudden or acute insanity: first, the isolation of the patient in a quiet or darkened room; forced alimentation; the control of motor excitement; reducing febrile states by tepid baths; sustaining the strength; and above all the inducing of sleep-these to be the cardinal lines of our treatment.

In conclusion, I may add that insanity may be modified in a community by the inspiration of high ideals, by agencies which are beneficial to sobriety and self-respect. I mean spiritual or moral agencies. These kindle a spirit of fervour, sympathy, and right-mindedness among the roughest characters, and in the most crowded areas of our cities. They are means which are peculiarly within the reach of medical men, who have opportunities of furthering them such as are afforded to no other profession.

\section{AN INQUIRY INTO THE EXISTENCE OF TYPHOID FEVER IN BERMUDA.}

\author{
LANCELOT KLLROY, and F. W. HOOPER, \\ Staff Surgeon, $\mathrm{R} N$. Dispenser in the Royal Navy. \\ [Communicated by the Director-Genrral, R.N.]
}

THE following paper represents the results of an investigation carried out to determine a question of considerable general interest, namely, whether cases of locally-acquired typhoid fever ever occur in Bermuda. Medical officers, both of the army and navy, stationed in Bermuda often have occasion to enter cases as typhoid fever in their returns, but it is commors in the island to take the view that the disease does not exist. This opinion many officers of both services have been inclined to accept, and hence to regard the cases encountered as paratyphoid and not true typhoid.

In order, therefore, to settle the question, a series of tests were carried out in the naval laboratory at Bermuda, and to ensure thorough control three strains of undoubted Eberth and a pure culture of bacillus coli communis were obtained and put through the subjoined series of tests side by side with three strains of organism obtained from the spleens of three fatal cases of this disease dying in the Royal Naval Hospital at Bermuda. Details of post-mortem examinations, etc., are purposely given fairly fully.

The histories of all three cases show that their disease must have been contracted in Bermuda. One case died from toxaemia, one from perforation, and one from haemorrhage.

Sources of Cultures Tested.

Strain A-Bacillus coli communis Strain D-Bacillus typhosus

Supplied from laboratory of R.N. Hospital, Haslar, by the courtesy of Fleet. Surgeon Bassett-Smith.

Strain B-Bacillus typhosus from Supplied from military New York

Strain C-Bacillus typhosus from

Strain $\mathbf{F}$ England laboratory, Bermuda, by the courtesy of Captain Wanhill, R.A.M.C.

Strain $\mathrm{E}$

Obtained as stated the text

in

Strain F was obtained from spleen of J. O., aged 47 years, joiner, H.M. Dockyard, Bermuda.

Clinical History. - This man had lived in Bermuda nine months before falling sick on October $x g$ th, 1904 . He had been ailing about 14 days; his temperature was r $100^{\circ} \mathrm{F}$; he complained of headacheand general malaise; he had a strong alcoholic history. October 2 rst. Found to be delirious at his residence at Somerset, Bermuda; temperature $10_{3}^{\circ}$; removed 